\title{
Enhanced cultivation of Helicobacter pylori in liquid media
}

\author{
H X Xia, L English, C T Keane, C A O’Morain
}

\begin{abstract}
Aims-To evaluate a technique for culture of Helicobacter pylori in large quantities of liquid media and to determine the factors that could influence the results.

Methods-Fifteen clinical isolates of $H$ pylori and a reference strain of $H$ pylori NCTC11637 were used to evaluate a method to cultivate the organism in 100 ml liquid medium comprising brain heart infusion broth with $5 \%$ horse serum and $0.25 \%$ yeast extract. Tissue culture flasks containing the inoculated liquid medium were placed in a $\mathrm{CO}_{2}$ incubator with $5 \% \mathrm{CO}_{2}$ for 2 hours and then incubated in a shaking incubator at 120 rpm.

Results-All the clinical isolates and the reference strain grew in the broth, although only a moderate growth of the reference strain occurred. Inoculum size significantly influenced the kinetics of growth of $H$ pylori in the liquid medium. Vancomycin, nalidixic acid, and amphotericin $B$, used to suppress contamination, did not affect growth of $H$ pylori in the medium. $\mathrm{CO}_{2}$ was essential for $H$ pylori to grow or survive in the liquid medium. Incubation with $\mathrm{CO}_{2}$ in a $\mathrm{CO}_{2}$ incubator for 30 minutes or 2 hours did not affect the results.

Conclusions-H pylori can be cultivated in large quantities of brain heart infusion broth with $5 \%$ horse serum and $0.25 \%$ yeast extract. Initial inoculum concentrations influence the kinetics of $H$ pylori growth in the liquid medium. Vancomycin, nalidixic acid, and amphotericin $B$ can be used as selective antimicrobial agents. $\mathrm{CO}_{2}$ is essential for initial growth of $H$ pylori in liquid media. The findings in this study may provide a useful, reproducible, and simple method for biochemical, molecular, and physiological studies of $H$ pylori, when those require large quantities of the organism.
\end{abstract}

(F Clin Pathol 1993;46:750-753)

Helicobacter pylori colonises human gastric epithelium and causes acute and chronic gastritis. ${ }^{1}$ It also has an important role in the development and recurrence of peptic ulcer disease. ${ }^{2}$ Isolation and subculture of $H$ pylori on various selective and non-selective solid media have been established. ${ }^{3-7}$ Growth of this organism in small amounts of liquid media has been achieved. ${ }^{45-13}$ Cultivation of $H$ pylori in large quantities of liquid media, however, essential for biochemical and physiological studies, is rarely reported. ${ }^{14-16}$ The aim of this study was to evaluate a method for the culture of $H$ pylori in large quantities of liquid media and to determine the factors that may influence the results.

\section{Methods}

In initial experiments a clinical isolate of $H$ pylori IRL92589 was used to establish suitable culture conditions. Three other clinical isolates of $H$ pylori, IRL92587, IRL92661, IRL92664, and a reference strain NCTC 11637 National Collection of Type Cultures, Public Health Laboratory, London, England) were added in a formal experiment for evaluation of reproducibility and feasibility of this method. Eleven other clinical isolates were also used. The isolate IRL92589 was used in all of the studies.

Solid media were used for growth of strains before inoculation of liquid media. $H$ pylori isolates and strain NCTC 11637 were subcultured on chocolate agar plates (Columbia agar base, LAB M, Topley House, Wash Lane, Bury, BL9 6AU, England) with 7\% horse blood. The plates were incubated in gas jars at $37^{\circ} \mathrm{C}$ for three days immediately after flushing with $\mathrm{CO}_{2}$. Colonies were examined using the urease test and Gram stain for confirmation of identity.

Liquid media used for a series of studies were based on brain heart infusion broth (Oxoid, Unipath Ltd, Basingstoke, Hampshire, England) with 5\% horse serum (Oxoid) and $0.25 \%$ yeast extract (Oxoid). Cultures from chocolate agar plates were inoculated into $5 \mathrm{ml}$ aliquots of nutrient broth to achieve graded concentrations of bacterial cells, determined by comparator turbidity tubes (McFarland No $0.5-$ No 5 ). Each suspension $(1 \mathrm{ml})$ was transferred to a 200 millilitre tissue culture flask (Sterilin Ltd, Hounslow, Middlesex) containing $100 \mathrm{ml}$ prewarmed liquid medium. The culture flasks were shaken gently and placed in a $\mathrm{CO}_{2}$ incubator (with $5 \% \mathrm{CO}_{2}$ ) for 2 hours with loosely fitted caps. The flasks were removed, caps tightened, and then incubated on a shaking platform (Model G25, New Brunswick Scientific Co. Inc. Edison, New Jersey, USA) at an angle of about $20^{\circ}$ with a rotation speed of $120 \mathrm{rpm}$. The cultures were examined daily for viable cell counts, and the urease test 


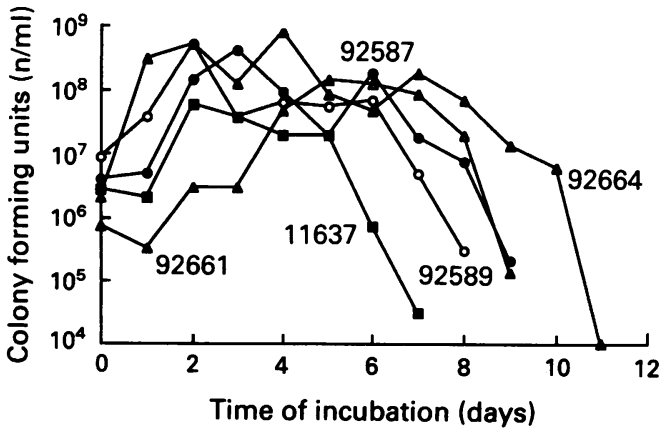

Figure 1 Growth of four clinical isolates and a reference strain NCTC 11637 in the liquid medium.

and Gram stain were also used. Oxidase and catalase tests were also used to outrule contamination.

Dilutions of $10^{-1}, 10^{-3}$, and $10^{-4}$ were made from the tissue culture flasks by suspending the culture in nutrient broth. The suspensions were inoculated with a $1 \mu$ l calibrated loop on to chocolate agar plates in triplicate. The plates were incubated as described above and colonies counted after five days of incubation. The mean value of three readings was calculated and expressed as colony forming units per millilitre (cfu/ml).

Bacterial cells of isolate IRL92589 from chocolate agar were suspended into nutrient broth to a turbidity of McFarland No 4 . Four 10-fold dilutions were then made to yield five inoculum concentrations in the liquid media (from about $10^{3}$ to $10^{7}$ ). The suspensions were inoculated into the broth and incubated as described above.

A suspension ( $1 \mathrm{ml}$ ) (McFarland No 4) of isolate IRL92589 was inoculated into four culture flasks containing $100 \mathrm{ml}$ of the liquid medium and also $6 \mathrm{mg} / 1$ vancomycin (Sigma Chemical Co. Ltd, USA), $20 \mathrm{mg} / 1$ nalidixic acid (Sigma), $4 \mathrm{mg} / \mathrm{l}$ amphotericin B (Sigma), or no antimicrobial agent, respectively. The culture flasks were incubated as described above.

Another suspension ( $1 \mathrm{ml}$ ) (McFarland No 4) of isolate IRL92589 was inoculated into three culture flasks. Subsequently, two flasks were kept in the $\mathrm{CO}_{2}$ incubator with loosely fitted caps for 30 minutes or 2 hours, respectively. The flasks then were removed and

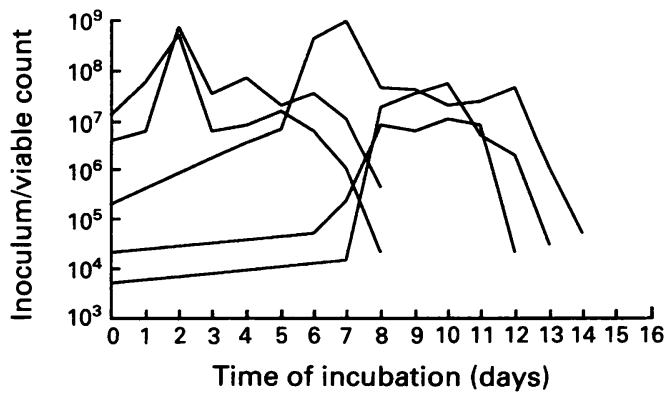

Figure 2 Growth of isolate IRL92589 with different inoculum concentrations in the liquid medium.

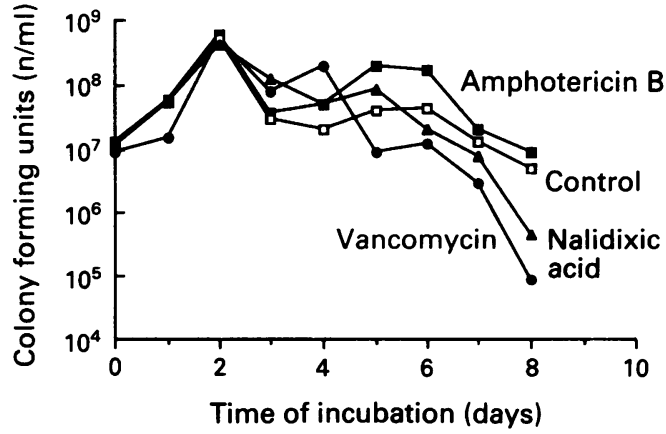

Figure 3 Effect of selective antibiotics on growth of isolate IRL92589 in the liquid medium.

incubated in the shaker at $120 \mathrm{rpm}$ as described before. The third flask was placed in the shaking incubator without $\mathrm{CO}_{2}$, as a control.

\section{Results}

In the initial study $H$ pylori isolate IRL92589 grew well in a tissue culture flask containing $100 \mathrm{ml}$ brain heart infusion broth supplemented with $5 \%$ horse serum and $0.25 \%$ yeast extract. This was when the flask was exposed to $5 \% \mathrm{CO}_{2}$ for about 2 hours and shaken at $120 \mathrm{rpm}$ at $37^{\circ} \mathrm{C}$. Subsequently, three other clinical isolates and a reference strain were evaluated in the same manner. The results for the five strains are shown in fig 1 . The reference strain NCTC11637 performed less satisfactorily in terms of the maximum growth achieved than the clinical isolates, all of which reached $10^{8} \mathrm{cfu} / \mathrm{ml}$ during the period of incubation. All the other 11 clinical isolates grew well in the medium (data not shown).

The five inoculum concentrations were used to determine the kinetics of growth of $H$ pylori. Different log phases of growth and different survival times appeared according to different inoculum concentrations (fig 2). With inocula of $10^{6}$ and $10^{7} \mathrm{cfu} / \mathrm{ml}$, maximum growth was achieved after two days of incubation; maximum growth was delayed when the inoculum concentrations decreased. With an inoculum of $10^{3} \mathrm{cfu} / \mathrm{ml}$, maximum growth occurred after 10 days of incubation. The survival times of $H$ pylori were shorter with the increased inoculum concentrations. With inocula of $10^{6}$ and $10^{7} \mathrm{cfu} / \mathrm{ml}$, the viable counts dropped below $10^{4} \mathrm{cfu} / \mathrm{ml}$ after nine days of incubation, while with inocula of $10^{3}$ $10^{5} \mathrm{cfu} / \mathrm{ml}$, the times were 13 to 15 days.

Vancomycin, nalidixic acid, and amphotericin B did not influence the growth of $H$ pylori in the liquid medium (fig 3). The pattern of growth in fig 3, showing two peaks, is similar to that in fig 2 . With inoculum of $10^{7}$ $\mathrm{cfu} / \mathrm{ml}$, the first peak of growth appeared after two days of incubation and the second after six days.

Incubation with $\mathrm{CO}_{2}$ for 30 minutes and 2 hours did not affect the growth of $H$ pylori in the liquid medium. Without incubation in $\mathrm{CO}_{2}$, however, $\mathrm{H}$ pylori survived in this 


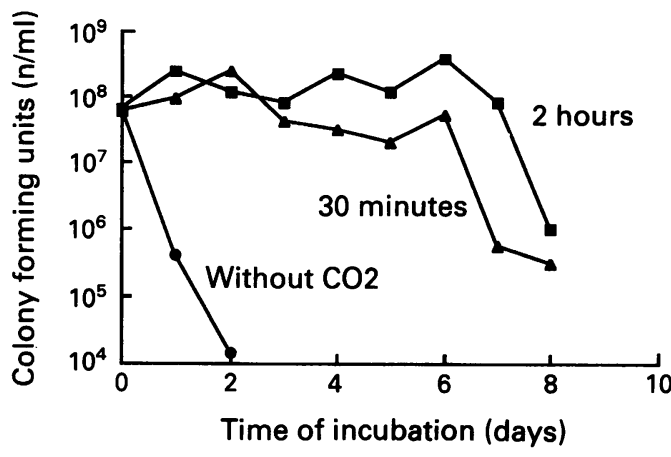

Figure 4 Effect of incubation time in $\mathrm{CO}_{2}$ on growth of isolate IRL92589 in the liquid medium.

medium for less than two days and viable bacterial cells were decreased considerably (fig 4).

\section{Discussion}

$H$ pylori is a microaerophilic organism which does not grow in poor conditions. Many methods for the isolation and subculture of this bacterium on different solid media have been established, but cultivation of this organism in broth, especially to achieve large quantities, still presents problems. Previous studies have shown that growth of $H$ pylori is achieved in Brucella broth supplemented with $1 \%$ to $10 \%$ fetal calf serum. ${ }^{12}{ }^{14}$ Fetal calf serum or horse serum is essential for optimal growth in tryptone soya broth, brain heart infusion broth, and Mueller-Hinton broth, whether in small or large volumes of liquid media. Tissue culture flasks were first applied by Shadowen $e t a^{l^{13}}$ to culture the organism in a biphasic system (an agar slant with overlying broth in a 2:1 volume ratio, the flask being incubated horizontally), but this system is unsuitable for large volume growth of $H$ pylori and is more applicable to recovery of $H$ pylori from clinical material. Shahamat et al reported a method for growth of large quantities $(600 \mathrm{ml})$ of $H$ pylori by several cycles of reincubation in tissue flasks (at least five times, four days each), either by transferring the cultures into new flasks containing an additional volume of liquid media or by adding a measured amount of the media into the flasks containing the cultures, and finally transferring these into $1-\mathrm{L}$ flasks. ${ }^{14}$ Unfortunately, the authors did not give the results of their method for large volume cultures. Drawbacks of this method are the prolonged procedure, cumbersome technique, and the hazard of contamination during the reinoculations. Hudson et al described a continuous culture technique using a chemostat apparatus, which is more technically demanding, thus limiting its routine use. ${ }^{15}$ Secker et al also successfully applied gas-permeable Lifecell tissue culture flasks for large quantity cultivation of $H$ pylori. ${ }^{16}$ Contamination is a hazard during the multiple samplings required unless performed in a laminar flow cabinet. In the present study all the 15 clinical isolates and a reference strain of $H$ pylori grew well in the
" $\mathrm{CO}_{2}$-incubated" tissue culture flasks, each containing $100 \mathrm{ml}$ brain heart infusion supplemented with $5 \%$ horse serum and $0.25 \%$ yeast extract, despite a relatively low growth of the reference strain NCTC11637. With this method, maximum growth of $6.2 \times 10^{7}$ to $5 \times 10^{8}$ was achieved after two to three days (fig 1), which was comparable with those obtained by other methods. ${ }^{12-16}$ Moreover, the method described above is simpler and easier to perform. It can give the required quantities of bacterial growth in a few days and contamination is not a hazard. This method may therefore provide a simple, reproducible method to produce large quantities of $H$ pylori.

To our knowledge this is the first report which has assessed $H$ pylori growth in liquid media for a prolonged period of time during which the viable count of the organism dropped to lower than $1 \times 10^{4} \mathrm{cfu} / \mathrm{ml}$, thus allowing us to examine the kinetics of $H$ pylori growth in the liquid media more completely. Based on the data obtained, $H$ pylori strains grew and survived in the medium tested for at least seven to 14 days (a viable count lower than $1 \times 10^{4} \mathrm{cfu} / \mathrm{ml}$ after several days of incubation usually meant that the organism did not survive; data not shown). Many factors might contribute to this, but the initial inoculum had an important role. Inoculum concentrations, as expected and reported, ${ }^{14}$ influenced the kinetics of growth of $H$ pylori. The greater the inoculum concentration, the shorter the time to the maximum growth, and the shorter the survival time of $H$ pylori in the medium (fig 2). Inocula of $10^{6}-10^{7} \mathrm{cfu} / \mathrm{ml}$ were found to be the optimal because these produced the maximum growth in the shortest time. In our experience the addition of 1 $\mathrm{ml}$ of suspension in nutrient broth with a turbidity equal to McFarland No 2 to No 3 into $100 \mathrm{ml}$ brain heart infusion was the optimal inoculum. If cultivation of the organism for more than eight days was needed, however, an appropriate lower inoculum should be used.

Cultivation of $H$ pylori in liquid media is difficult, partly because of contamination. Selective solid media with vancomycin, amphotericin B, and nalidixic acid are commonly used in the isolation of $H$ pylori from gastric biopsy specimens. ${ }^{451012}$ Vancomycin and amphotericin B are also used in broth cultures to control contamination. ${ }^{1316}$ Their potential effect on growth of the bacterium, however, has not been determined. This study showed that vancomycin and amphotericin $\mathbf{B}$, as well as nalidixic acid, did not affect growth of $H$ pylori in the liquid medium (fig 3), suggesting that these antimicrobial agents can be used alone or in combination to suppress contaminating micro-organisms.

$\mathrm{CO}_{2}$ is essential for primary isolation of $\mathrm{H}$ pylori from gastric biopsy specimens. This organism, however, can grow on non-selective solid media in an aerobic atmosphere with high humidity once isolated from gastric biopsy specimens (authors' unpublished observations). Previous studies suggested that 
$10 \% \mathrm{CO}_{2}$ is required for the growth of $\mathrm{H}$ pylori in liquid media. ${ }^{12} 14$ In the present study $\mathrm{CO}_{2}$ was confirmed to be necessary to cultivate $H$ pylori in the liquid medium and it must be supplied in an even distribution throughout the broth by placing the flask almost horizontally and shaking the flask at $120 \mathrm{rpm}$ during incubation (fig 4). It was found, however, that $5 \% \mathrm{CO}_{2}$ was sufficient for growth of $H$ pylori and could be obtained by placing the loosely capped tissue culture flask containing inoculated medium in a $\mathrm{CO}_{2}$ incubator with $5 \% \mathrm{CO}_{2}$ for only 30 minutes and then tightening the caps.

In conclusion, $H$ pylori can be cultivated in large quantities of brain heart infusion broth supplemented with $5 \%$ horse serum and $0.25 \%$ yeast extract. Initial inoculum concentrations influence the kinetics of growth of $H$ pylori in the liquid medium. Vancomycin, nalidixic acid, and amphotericin B do not affect growth of the organism in broth, and can be used as selective antimicrobial agents if contamination is a hazard. $\mathrm{CO}_{2}$ is essential for initial growth of $H$ pylori in liquid media and a concentration of $5 \%$ is sufficient. This can easily be obtained by placing a tissue culture flask of the inoculated medium in a $\mathrm{CO}_{2}$ incubator containing $5 \% \mathrm{CO}_{2}$ for 30 minutes. The findings of this study may provide a useful, reproducible, and simple method for biochemical, molecular, and physiological studies of $H$ pylori, when these require large quantities of the organism.

1 Marshall BJ, Warren, JR. Unidentified curved bacilli on gastric epithelium in active chronic gastritis. Lancet
1983; i:1273-5.

2 Patchett S, Beattie S, Leen E, Keane C, O'Morain C. Helicobacter pylori and duodenal ulcer recurrence. $\mathrm{Am}$ $\mathcal{F}$ Gastroenterol 1992;87:24-7.

3 Ansorg R, Recklinghausen GV, Pomarius R, Schmid EN. Evaluation of techniques for isolation, subcultivation, Evaluation of techniques for isolation, subcultivation,
and preservation of Helicobacter pylori. $f$ Clin Microbiol 1991;29:51-3.

4 Buck GE, Smith JS. Medium supplement for growth of Campylobacter pyloridis. $f$ Clin Microbiol 1987;25: 597-9.

5 Goodwin CS, Blincow ED, Warren JR, Waters TE, Sanderson CR. Evaluation of cultural techniques for isolating Campylobacter pyloridis from endoscopic biopsies of gastric mucosa. F Clin Pathol 1985;38: 1127-31.

6 Hazell SL, Markesich DC, Evans DJ, Evans DG, Graham DY. Influence of media supplements on growth and survival of Campylobacter pylori. Eur $\mathfrak{f}$ Clin Microbiol survival of Campylobacter

7 Mathai E, Cafferkey M, Keane C, O'Morain C. Evaluation of different solid media used for cultivation of Helicobacter (Campylobacter) pylori. Indian $\mathcal{F} \mathrm{Med}$ 1992;95:23-5.

8 Dent JC, McNulty CAM. Evaluation of a new selective medium for Campylobacter pylori. Eur $₹$ Clin Microbiol Infect Dis 1988;7:555-68.

9 Goodwin CS, Blake P, Blincow E. The minimum inhibitory and bactericidal concentrations of antibiotics and anti-ulcer agents against Campylobacter pyloridis. $\mathcal{F}$ Antimicrob Chemother 1986;17:309-14.

10 Krajden S, Bohnen J, Anderson J, et al. Comparison of selective and nonselective media for recovery of Selective and nonselective media for recovery of Campylobacter pylori from

11 Leunk RD, Johnson PT, David BC, Kraft WG, Morgan DR. Cytotoxic activity in broth-culture filtrates of Campylobacter pylori. $\mathcal{F}$ Med Microbiol 1988;26:93-9.

12 Morgan DR, Freedman R, Depew CE, Kraft WG. Growth of Campylobacter pylori in liquid media. $f$ Clin Microbiol 1987;25:2123-5.

13 Shadowen RD, Sciortino C. Improved growth of Campylobacter pylori in a biphasic system. I Clin Campylobacter pylori in
Microbiol $1989 ; 27: 1744-7$.

14 Shahamat M, Mai UEH, Paszko-Kolva C, Yamamoto $H$ Colwell RR. Evaluation of liquid media for growth of Colwell RR. Evaluation of liquid media for growth

15 Hudson MJ, Newell DG. Continuous culture of Campylobacter pylori. In: Megraud F and Lamouliatte $\mathrm{H}$, eds. Gastroduodenal pathology and Campylobacter pylori. Amsterdam: Elsevier Science Publishers BV, 1989:11-15.

16 Secker DA, Tompkins DS, Alderson G. Gas-permeable Lifecell tissue culture flasks give improved growth of Helicobacter pylori in a liquid medium. $\mathcal{F}$ Clin Microbiol 1991;29:1060-1. 